\title{
SEMANTIC CONDITIONING IN INTERACTION AND TRANSMISSION
}

\author{
OLGA FEHÉR $^{* 1}$, ELIZABETH WONNACOTT ${ }^{2}$, HANNA JÄRVINEN ${ }^{3}$, KENNY $^{2}$ \\ $\mathrm{SMITH}^{3}$ \\ *Corresponding Author: o.feher@warwick.ac.uk \\ ${ }^{1}$ Psychology Department, University of Warwick, Coventry, United Kingdom \\ ${ }^{2}$ Division of Psychology and Language Sciences, University College London, \\ London, United Kingdom \\ ${ }^{3}$ Linguistics and English Language, Edinburgh, United Kingdom
}

A central question in language evolution research is how fundamental properties of language have evolved and how that evolutionary process is shaped by human cognition. One property observed in all natural languages is variation. Linguistic variation tends not to be random and fully unpredictable. Rather, it is conditioned on the linguistic or social environment (Givón, 1985): linguistic or social context deterministically or probabilistically predicts the use of linguistic variants.

Previous research has shown that when children acquire artificial languages containing unpredictable variation, they often eliminate the variation by overusing one of the variants (e. g. Hudson Kam \& Newport, 2009). However, at present there is no satisfying experimental account of why natural languages should contain so much conditioned variation or how conditioning comes about. We investigated the evolution of conditioned variation using an artificial language paradigm that included transmission and interaction.

We presented participants with images of objects accompanied by their descriptions in an artificial language. Depending on experimental condition, the objects were drawn from either one semantic category (e.g. all objects were animals) or two semantic categories (a mix of animals and vehicles). Each description consisted of a nonsense verb, a noun for the object and, for scenes involving multiple objects, a variable plural marker. The plural was marked by one of two markers (e.g. dak and fip) which occurred equally frequently in our initial experimenter-designed languages. 
After training, participants were first asked to produce the descriptions they learnt for images (recall phase), and then they used the language to play a communication game with another participant or a simulated partner (interaction phase). We measured the use of the plural markers during recall and interaction, and in particular tracked whether variation was preserved in the miniature language, became conditioned (on lexical or semantic context) or was eliminated (by one marker overtaking the other).

In Experiment 1 we looked at the consequences of transmission for the evolution of conditioned variation. Participants were trained and tested on an artificial language as described above; we then took the recall data from one participant and used it as the training data for another participant, in a standard Iterated Learning paradigm. Conditioned variation evolved through this transmission process: in One Category chains (i.e. where stimuli were drawn from one semantic category) variation became lexically conditioned, whereas in Two Category chains, variation became conditioned on semantic category (i.e. animals took one marker, vehicles the other).

In Experiment 2 we ran transmission chains where each generation consisted of two participants, whose combined output was used as input for the next generation. In one condition, we used their recall data as input. Stable conditioned variation did not evolve here: in both One Category and Two Category chains the mixing of output from multiple independent individuals blocked any cumulative conditioning, replicating the findings of Smith et al. (2017). In another condition, the combined output produced by each pair during interaction (rather than recall) formed the input to the next generation. Although interaction allowed pairs to converge on a shared system, conditioned variation still did not evolve: One Category chains tended to eliminate one of the two variants, whereas Two Category chains retained both variants in free variation.

Finally, in Experiment 3 we imposed a bottleneck on transmission: participants were tested on novel nouns, forcing them to generalise their system of plural marking. Although semantically-conditioned systems of variation would allow generalisation, they did not evolve in transmission chains featuring more than one participant per generation; rather, variation was gradually eliminated.

These findings suggest that individual learners possess a bias for semantic conditioning (as observed in Experiment 1), but this bias does not straightforwardly produce stable semantically-conditioned patterns of variation; rather, systems lacking in variability are generally preferred. The conditions under which the patterns of conditioned variation we see in natural language therefore remain to be identified. 


\section{References}

Givón, T. (1985). Function, structure, and language acquisition. In D. Slobin (Ed.). The crosslinguistic study of language acquisition (pp. 1005-1028). Hillsdale, NY: Lawrence Erlbaum.

Hudson Kam, C. L. \& Newport, E. L. (2009). Getting it right by getting it wrong: When learners change languages. Cognitive Psychology, 59, 30-66.

Smith, K., Perfors, A., Fehér, O., Samara, A., Swoboda, K., \& Wonnacott, E. (2017). Language learning, language use and the evolution of linguistic variaiton. Philosophical Transactions of the Royal Society B, 372, 20160051. 